\title{
Lymphome non hodgkinien B mandibulaire isolé révélé par une hypoesthésie labio- mentonnière et un élargissement du canal mandibulaire
}

\author{
Chbicheb S, Hakkou F, El Wady W \\ Service d'Odontologie chirurgicale, Université Mohammed V Souissi \\ Rabat, Maroc
}

Les lymphomes correspondent à des proliférations malignes intéressant le tissu lymphoïde; ils sont principalement constitués par des lymphomes non hodgkiniens (LNH). Le tube digestif, avec la cavité buccale, représente le principal site des LNH extra-ganglionnaires; la mandibule est plus fréquemment atteinte que le maxillaire. Selon la classification de l'OMS, on distingue les LNH peu évolutifs, les LNH agressifs (type DLBCL ou diffuse large B cell lymphoma) et les LNH hautement agressifs (lymphome de Burkitt); le sous-type DLBCL représente 58\% des cas de lymphomes (Kemp et al. 2008).

Une femme de 35 ans a consulté pour des douleurs hémi-mandibulaires gauches, avec des épisodes d'hypoesthésie labiale homolatérale. Un signe de Vincent s'est progressivement installé. Cliniquement, on a noté la présence d'une tuméfaction dure dans le vestibule inférieur gauche en regard de la 34. Le bilan radiographique (radiographie panoramique et CT-scan) a montré un élargissement du canal mandibulaire et du foramen mentonnier gauches. Une biopsie de la tuméfaction a été réalisée, sous anesthésie locale, en regard du foramen mentonnier; l'examen histologique et immunohistologique a établi le diagnostic de LNH de phénotype B. Le bilan d'extension comprenant un CT-scan corps entier, une échographie cardiaque avec FEVG (fraction d'éjection du ventricule gauche), un bilan biologique (numération formule sanguine, vitesse de sédimentation, ionogramme, lactate déshydrogénase, $\mathrm{BH}$, sérologie pour le VHB, le VHC et le VIH) et une biopsie ostéomédullaire n'ont pas mis en évidence d'autres localisations; il s'agissait d'un LNH de phénotype B, stade IAa localisé. La patiente a bénéficié d'un traitement associant 7 cures de chimiothérapie à base de R-CHOP (rituximab, cyclophosphamide, doxorubicine, vincristine et prednisolone) suivi d'une radiothérapie locale. La patiente est en rémission depuis une année; la sensibilité nerveuse est redevenue normale, l'élargissement du canal mandibulaire a disparu.

Les lymphomes primitifs de la mandibule sont rares, ils représentent environ 0,6\% des LNH extra-ganglionnaires selon Bachaud (1992). Ils surviennent à tout âge et ils sont plus fréquents chez l'homme. Ils se manifestent le plus souvent par une tuméfaction indolore, éventuellement ulcérée dans la cavité buccale. La confusion initiale avec une pathologie d'origine dentaire est fréquemment rapportée. Le diagnostic est exclusivement histopathologique. Le recours aux colorations immunohistochimiques à la recherche de marqueurs spécifiques et à la microscopie électronique est souvent indispensable. Lorsque le diagnostic est établi, un bilan d'extension s'impose pour éliminer une ou plusieurs localisations secondaires. Le traitement associe de façon variable radiothérapie et/ou chimiothérapie. Le pronostic des lymphomes osseux est plus favorable que celui des autres tumeurs malignes osseuses, avec un taux de survie à 5 ans de 40-50\% (Trost et al. 2008).

Correspondance : s_chbicheb@yahoo.fr 